\title{
Ökonomisierung aus der Sicht der Online-Medien
}

\author{
Josef Trappel
}

Online-Medien sind zu einer eigenständigen Mediengattung berangewachsen, die nicht nur die klassischen Merkmale von Massenmedien aufweist, sondern über diese hinausgebt. Multimedialität und direkte Interaktion erweitern das Leistungsspektrum der Online-gegenüber traditionellen Medien. Der Beitrag geht der Frage nach, welche Formen der Ökonomisierung und Kommerzialisierung in dieser neuen Mediengattung zu beobachten sind. Dabei wird zunächst eine begriffliche Unterscheidung vorgenommen. Die Analyse der einschlägigen Wertschöpfungskette zeigt, dass die Betreiber von Online-Medien aufgrund der empfangsseitigen Plattformvielfalt zur Adaptierung ibrer Inhalte gezwungen sind. Die Digitalisierung der Inhalte ermöglicht die Herstellung endgeräteabbängiger „Versionen“, verfübrt aber auch zur Mehrfachverwertung von Inhalten, obne den publizistischen Wert zu erböhen. Der Beitrag kommt zum Schluss, dass die bisher beobachteten Erscheinungsformen der Online-Medien einen hohen Kommerzialisierungsgrad aufweisen und die Tendenz zur Ökonomisierung beschleunigen.

Im vorwissenschaftlichen Verständnis und im herkömmlichen medialen Diskurs wandelt sich das Image des Internets. Nicht lange ist es her, da galt das Netz der Netze zu allererst als Eldorado des schöpferischen Chaos, als Selbstverwirklichungsarena kommunikativ benachteiligter Gruppen und als listige Revanche technisch versierter Habenichtse über das dröge Medienestablishment. Heute überwiegt bereits das Image als E-Commerce-Plattform und - im Medienbereich - als verlängerte Werkbank für bereits anderswo vermarktete Inhalte.

Ein strukturierter Blick auf die neu entstehenden Medien- und Kommunikationsformen bestätigt die Annahme, das Internet sei ein durchkommerzialisierter Interaktionsraum, dessen Regeln von dominierenden Akteuren bestimmt werden. Der selbst organisierte Bereich, der auch in der analogen Welt die etablierten Massenmedien meist unhörbar, gelegentlich aber auch lautstark begleitet, sieht sich im Internet in die Defensive gedrängt. Dafür zeichnen nicht zuletzt ökonomische Mechanismen verantwortlich, die aus dem analogen Wirtschaftsleben bestens bekannt sind. Ökonomisierung und Kommerzialisierung prägen dem jungen Medium längst ihre Stempel auf.

Aus publizistikwissenschaftlicher Perspektive sind jene Teilbereiche des Internets von besonderem Interesse, die explizit oder implizit mediale Funktionen erfüllen und daher als Online-Medien zu bezeichnen sind. Der Gegenstand der folgenden Betrachtung bleibt daher auf diejenigen Internetsektoren beschränkt, in denen publizistisch relevante Leistungen erbracht werden. Zu den Auswirkungen der Ökonomisierung auf solche Online-Medien sollen drei Thesen geprüft werden:

- These 1: Bei den Online-Medien erweist sich die Ökonomisierung als dominierendes Gestaltungsprinzip.

- These 2: Mit Ausnahme hochpreisiger Nischenprodukte übersteigt der Kommerzialisierungsgrad der Online-Medien denjenigen der analogen Medien.

- These 3: Aufgrund der bei Online-Medien deutlich ausgeprägten Gesetzmäßigkeiten der „New Economy“ nimmt die Marktmacht führender Marktteilnehmer weiter zu. Um die Folgen der Ökonomisierung für die Online-Medien bzw. deren Beitrag zur Ökonomisierung der Medien näher zu untersuchen, bedarf die verwendete Begrifflichkeit der näheren Erläuterung. 


\section{1. Ökonomisierung und Kommerzialisierung}

Für die Analyse der Auswirkungen der Ökonomisierung auf die Online-Medien erscheint eine begriffliche Unterscheidung von Ökonomisierung und Kommerzialisierung hilfreich. Unter Ökonomisierung sei hier die Tendenz verstanden, das wirtschaftliche Handeln im Bereich der Medien immer konsequenter an einem Marktziel (z. B. Einkommensmaximierung) zu Lasten nicht-marktlicher (z. B. publizistischer) Ziele auszurichten. Diese Tendenz lässt sich sowohl bei analogen Medien als auch im Bereich der digitalen Online-Medien beobachten und belegen. Sie ist also keineswegs ein neues Phänomen im Medienbereich. Ob durch die Digitalisierung diese Tendenz allenfalls eine Beschleunigung erfährt, soll in diesem Beitrag diskutiert werden.

Im Gegensatz zur Ökonomisierung bezeichnet der Begriff der Kommerzialisierung hier die einfache betriebswirtschaftliche Tatsache, dass ein immer größerer Erlösanteil auf indirektem Weg erzielt wird. Mit dem Grad der Kommerzialisierung nimmt also der Anteil jener Erlöse ab, die als direktes Entgelt für den Konsum der medialen Dienstleistung entrichtet werden. Auch diese Tendenz lässt sich in der analogen Medienwelt beobachten, in extremer Ausprägung etwa bei den Gratiszeitungen („freesheets“), die in immer mehr europäischen Ballungsräumen zum Konsum, nicht aber zum Kauf angeboten werden. Auch das frei empfangbare kommerzielle Fernsehen weist den höchsten möglichen Kommerzialisierungsgrad auf.

Die beiden Begriffe bezeichnen zwei unterschiedliche Sachverhalte. Der Ausprägungsgrad von Ökonomisierung und Kommerzialisierung bei Online-Medien ist Gegenstand des vorliegenden Beitrags.

\section{Kennzeichen von Online-Medien}

Die im Internet und World Wide Web abgebildete virtuelle Realität hat einen Komplexitätsgrad erreicht, der eine strikte Eingrenzung des Untersuchungsgegenstands nahe legt. $\mathrm{Zu}$ verschieden sind Motivation, Kommunikationsziel und Gestaltung der Angebote im Internet, als dass alle Websites generell dem Mediensektor zuzuschlagen wären. Ebenso wenig wie in der ,analogen “ Kommunikationswelt Warenhausprospekte, Postwurfsendungen und Geschäftsberichte den Massenmedien zuzurechnen sind, sind E-Commerce-Angebote, Marketing-Mailings und Unternehmens-PR im Internet ihrem Charakter nach als Medien zu qualifizieren.

Im World Wide Web verdient aber jener Sektor publizistikwissenschaftliche Aufmerksamkeit, der sich durch medienähnliche Dienstleistungen auszeichnet. Solche „Online-Medien“ erfüllen zunächst die klassischen Definitionsmerkmale von Massenmedien. Sie sind öffentlich (potenziell für jedermann empfangbar), technisch vermittelt, richten sich an ein disperses Publikum, zeichnen sich durch Periodizität aus und weisen einen Gegenwartsbezug auf. Online-Medien weisen darüber hinaus aber noch weitere konstitutive Merkmale auf. Sie sind multimedial (sie überschreiten die medialen Grenzen, die den Print- und elektronischen Medien gesetzt sind), sie sind digital (und daher empfangsseitig verarbeit- und verbreitbar) und sie sind interaktiv (gestatten die direkte Rückkopplung) (vgl. Sennwald 1998, 9f.).

Unter diesem Gesichtspunkt bilden die Online-Medien eine eigenständige Mediengattung, deren spezifische Ausprägungen einem raschen Wandel unterliegen und deren Form sich kontinuierlich verändert. Für die Zwecke der vorliegenden Untersuchung bleibt der Gegenstand auf jene publizistisch relevanten Web-Angebote beschränkt, die den klassischen Merkmalen der Massenmedien entsprechen und die über reine Unter- 
nehmenskommunikation hinausgehen. Die Materialen der Unternehmens-PR (Newsletter, Mailinglists etc.) gelten damit nicht als Online-Medien und bleiben ausgeschlossen. Demgegenüber zählen neue Formen wie E-Zine, Webradio und Net-Zeitungen zu den Online-Medien.

Die solchermaßen eingegrenzten Online-Medien lassen sich zunächst in zwei Gruppen kategorisieren. Neben den Online-Ablegern bestehender Medien sind neue Unternehmen in den Markt eingetreten, die keine originäre Bindung zu klassischen Medien aufweisen. Zu der ersten rasch wachsenden Gruppe zählen die Online-Ausgaben der Zeitungen, Zeitschriften und Magazine (eine umfassende Liste deutschsprachiger Zeitungen ist zu finden unter http://www.zmg.de/homepage/Produkte_und_Services/Zeitungen_im_Internet/) sowie der Radio- und Fernsehveranstalter (jeweils unter deren Markennamen im Internet).

$\mathrm{Zu}$ der Gruppe der „neuen“ Online-Medien zählen nicht nur Start-up Companies wie beispielsweise die Netzeitung.de (operativ seit November 2000, nach dem Vorbild der norwegischen Nettavissen.no und mit norwegischem Know-how), sondern auch medienähnliche Angebote. Solche Angebote kommen häufig von Unternehmen, deren Geschäftszweck bislang nicht die Versorgung der Öffentlichkeit mit Information, Bildung oder Unterhaltung war. Beispielsweise hat die Schweizer Post auf ihrem Internet-Portal einen medienähnlichen Dienst eingerichtet (www.yellowworld.ch), der sich kaum noch von der Online-Ausgabe einer Tageszeitung unterscheidet. Dieses Portal wird von einer rund 40-köpfigen journalistischen Redaktion betreut, die angebotenen Inhalte sind aktuell und journalistisch aufbereitet und wenden sich an ein Massenpublikum.

Portale dieser Ausrichtung werden in unterschiedlicher inhaltlicher Tiefe von einer Vielzahl von Unternehmen, zumeist aus dem Dienstleistungssektor, angeboten. Banken, Versicherungen, Einzelhandelshäuser, aber auch direkt dem Internet selbst zuordenbare Unternehmen (Service Provider, Suchmaschinen) versuchen, durch aktuelle Beiträge ihren Internetauftritt aufzuwerten, Kunden zu gewinnen, „Traffic“ zu generieren und damit neue Erlösquellen zu erschließen.

Die Gruppe der „neuen“ Online-Medien besteht ihrerseits also erneut aus Angeboten, die von professionellen Journalistinnen und Journalisten mit massenmedialem Anspruch hergestellt werden, und solchen, die mediale Leistungen lediglich zur Erzeugung von Aufmerksamkeit für andere Dienstleistungen erbringen.

Hinzu kommt noch eine Ausprägung von Online-Medien, die den professionellen Standards von Massenmedien in der Regel nicht genügen. Solche „Nachrichtencommunities“ (wie z.B. www.shortnews.de) stellen auf eine gemeinsame Website ungefiltert und ungeprüft Nachrichten, die von Mitgliedern der Community zugeliefert werden. Das Resultat bezeichnen Beobachter als „Parajournalismus von Laien“ (Neuberger 2000, 310). Solche Laienmedien erfüllen in der Regel die Anforderungen an die branchenüblichen professionellen Standards (z. B. redaktionelle Bearbeitung nach journalistischen Grundsätzen) nicht. Sie stellen eine bisher nicht verfügbare Form der Massenkommunikation dar, deren äußere Form medienähnlich ist, deren innere (Organisations-)Struktur aber der Liebhaberei näher steht als professionell geführten Medienbetrieben. Sie bilden eine Sonderform der Online-Medien, die aufgrund der völlig unterschiedlichen Produktions-, Vermittlungs- und Organisationsstruktur von den weiteren Betrachtungen ausgeschlossen bleibt. 


\section{Wertschöpfungsketten der Medien im Wandel}

In ökonomischer Hinsicht weisen neue und ausgelagerte Online-Medien eine Reihe von Gemeinsamkeiten mit den herkömmlichen Medien auf. Online-Medien und herkömmliche Medien sind durch hohe Fixkosten und geringe variable Kosten charakterisiert (die Kosten für die Herstellung der Inhalte bleiben - unabhängig von der Anzahl der hergestellten Exemplare, der das Signal empfangenden Zuhörer/-seher oder der Internetnutzer - gleich), sie können durch Skaleneffekte Wettbewerbsvorteile generieren (höhere Auflagen, größere Reichweiten, zahlreichere Visits begünstigen den Marktführer überproportional und können Monopolisierungstendenzen nach sich ziehen), sie agieren in weitgehend gesättigten Märkten (neu in den Markt eintretende Wettbewerber müssen die Nutzungszeit/Aufmerksamkeit des Publikums größtenteils auf Kosten bestehender Wettbewerber erwerben) und ihr Verkaufspreis spielt eine vergleichsweise untergeordnete Rolle (bei Werbemedien trägt der Kaufpreis nur in Einzelfällen maßgeblich zum Gesamterlös bei) (zu den medienökonomischen Grundlagen vgl. Heinrich 1994 und 1999). Dennoch sind bei der Betrachtung der einschlägigen Wertschöpfungsketten Unterschiede zu erkennen.

\section{Abb. 1: Wertschöpfungskette klassischer Medien}

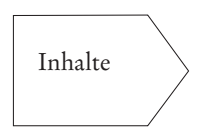

$\begin{array}{ll}\text { Produktion } & \text { Interaktion } \\ \text { Beschaffung } & \text { Organisation } \\ \text { Rechteerwerb } & \text { Eigentümer } \\ \text { Redaktion } & \text { Planung }\end{array}$

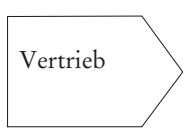

Ausstrahlung

Zustellung

Straßenverkauf

Einzelhandel

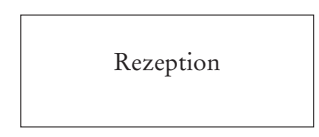

Endgeräte

Nutzungsgewohnheiten

Öffentlichkeit

In der Wertschöpfungskette klassischer Medien lassen sich die nachgelagerten Stufen den generierten Inhalten eindeutig zuordnen. Für die gedruckte Zeitung kommt nur Papier als Trägermedium in Frage, das durch ein ausgefeiltes Logistiknetz zu den Leserinnen und Lesern transportiert wird. Ebenso sind Radio- und Fernsehinhalte jeweils einem einzigen Empfangsgerät zuzuordnen, wobei das Sendesignal auf unterschiedliche Weise zu den Endgeräten transportiert wird (terrestrisch, Kabel, Satellit). In vielen Fällen kontrolliert der Medieneigentümer die gesamte Kette bis zu den Haushalten, ohne auf Partnerschaften angewiesen zu sein.

Online-Medien weisen grundsätzlich dieselbe Wertschöpfungskette auf, sie ist jedoch um einige Stufen erweitert (Abb. 2).

Neu schließt das „Packaging“ an die Stufe der Inhaltegenerierung an. Packaging bezeichnet die meist multimediale Bündelung von Inhalten. So ergänzen Webradio-Anbieter den aktuellen Musiktitel, der in Streaming-Technologie hörbar gemacht wird, durch Bild- und Textinformation über Interpret und Musikverlag und bieten so ihren Hörerinnen und Hörern ein zusätzliches Angebot. Ebenso sind in Net-Zeitungen Hörfiles enthalten, die beispielsweise den Originalton zu einem als Text vorliegenden Interview liefern. Oft werden Informationen aus unterschiedlichen Quellen zu solchen „Packages“ zusammengefasst.

Durch die Möglichkeit, einmal erzeugte Inhalte in unterschiedlichen Versionen mit 
Abb. 2: Wertschöpfungskette Online-Medien

\begin{tabular}{|c|c|c|c|c|c|}
\hline Inhalte & Packaging & $\begin{array}{l}\text { Verarbeitung } \\
\text { Wert- } \\
\text { erstellung }\end{array}$ & Vertrieb & Endgeräte & Rezeption \\
\hline Produktion & Inhaltebündel & Interaktion & Netzwerk & Fernsehen & Nutzungs- \\
\hline Beschaffung & Anpassung an & Organisation & Plattform & PDA/PC & gewohnheiten \\
\hline Rechteerwerb & Plattformen & Eigentümer & Partner & Internet & Öffentlichkeit \\
\hline Redaktion & Versioning & Planung & Portale & Mobiltelefon & Segmentierung \\
\hline
\end{tabular}

unterschiedlichen Qualitätsmerkmalen auf den virtuellen Markt zu bringen, erweitern Online-Medien - scheinbar - ihre Angebotsbreite. Beim „Versioning“ (Begriff von Shapiro/Varian 1998; vgl. auch Zerdick u. a. 1999, 187) etwa werden die Inhalte nicht vermehrt, sondern lediglich in plattformspezifischen Formen weiterverarbeitet. So kann beispielsweise ein redaktioneller Textbeitrag in voller Länge in einer Net-Zeitung erscheinen, in gekürzter Form für die Displays von DAB-Radios aufbereitet werden und als Kurzmeldung auf WAP-taugliche Mobiltelefone übermittelt werden. Dadurch nimmt die Angebotsbreite (Präsenz in mehreren Kanälen) zu, nicht jedoch die inhaltliche Vielfalt. Dennoch entsteht durch die Kompatibilität der Inhalteversionen mit den Endgeräten jener Mehrwert (Nutzen), der schließlich Userinnen und User zum Konsum solcher Leistungen veranlasst.

Die zweite neue Stufe sind die Endgeräte. Der Konsum von Online-Medien setzt die Verfügbarkeit tauglicher Endgeräte zwingend voraus. Solche Endgeräte sind aber den Online-Medien nicht direkt zuordenbar, so wie das Fernsehgerät dem Fernsehangebot zuordenbar ist. Vielmehr steht für die Nutzung von Online-Medien eine Vielfalt von Endgeräten zur Verfügung, jedes einzelne mit höchst unterschiedlichen spezifischen Merkmalen. Nicht einmal die Existenz eines Bildschirms kann vorausgesetzt werden (z. B. MP3 Player). Daher wird die Auswahl der bedienten Funktionalitäten möglicher Empfangsgeräte zur strategischen unternehmerischen Entscheidung. Mit dieser Entscheidung sind Fragen der Kompatibilität der Hard- und Software ebenso verknüpft wie diejenige nach der Offenheit der eingesetzten Standards. Endgeräte, in der analogen Wertschöpfungskette noch der Stufe Rezeption zugeordnet, werden für Online-Medien also erheblich aufgewertet.

Die Wertschöpfungsstufe „Endgeräte“ entzieht sich der Kontrolle durch die OnlineMedien. Ihr Einfluss auf Design und Funktionalität von PCs, Laptops, Subnotebooks, PDAs, Mobiltelefonen und UMTS-Empfangsgeräten ist gering. Online-Medien müssen also die erforderliche Flexibilität aufbringen, ihr Angebot kontinuierlich den Vorgaben der Hardware-Industrie anzupassen.

Online-Medien sind also, um ihr Publikum zu erreichen, stärker als ihre analogen Vorfahren von technologischen Systementscheidungen abhängig. Analoge Fernsehund Radioveranstalter können zwar produktionsseitig ihr Angebot mit den neuesten Technologien ausstatten, empfangsseitig ist das technische Format seit Jahrzehnten vorgegeben. Online-Medien hingegen sind mit ständig wechselnden Empfangsgeräten mit unterschiedlichsten Standards konfrontiert, an die das Inhalteangebot kontinuierlich angepasst werden muss.

Wichtiger als die Verlängerung der Wertschöpfungskette durch zusätzliche Stufen sind im Hinblick auf die Ökonomisierung und Kommerzialisierung der Online-Medien die qualitativen Veränderungen auf jeder einzelnen Stufe. 
Inhalte und Packaging: Ausgelagerte Online-Medien weisen einen hohen Grad an inhaltlicher Übereinstimmung mit den analogen Ausgangsmedien auf. In einer vergleichenden Untersuchung von Papier- und Online-Ausgaben deutschsprachiger Zeitungen kommt Zürn zum Resultat, dass die inhaltliche Breite der Online-Ausgabe nicht mit derjenigen der Printausgaben Schritt hält. Zwischen 33\% und 85\% der Artikel der Printausgaben fanden sich im Internet wieder, exklusive Online-Inhalte fanden sich so gut wie nie. „In keiner der drei untersuchten Zeitungen konnte ein nennenswerter Anteil an reinen Onlineartikeln nachgewiesen werden“ (Zürn 2000, 320). Dieses Resultat legt den Schluss nahe, dass in dieser noch relativ frühen Entwicklungsphase die printbezogenen Online-Medien zum überwiegenden Teil durch fremdgenerierte Inhalte gespeist werden. Für die „neuen“ Online-Medien liegen diesbezüglich noch keine Untersuchungsergebnisse vor.

Die Inhalte der Online-Versionen der elektronischen Medien Radio und Fernsehen nehmen in der Regel engen Bezug auf das ausgestrahlte Programm bzw. die anvisierten Zielgruppen. Eine erste Sichtung der einschlägigen Websites zeigt, dass Fernsehveranstalter zwei unterschiedliche Produktstrategien im Netz verfolgen: Für die eine Gruppe bildet das ausgestrahlte Programm die inhaltliche Leitlinie, je um zielgruppenspezifische Features ergänzt (z. B. Websites der ARD-Anstalten; Online-Spiele für Unterhaltungsprogramme wie bei sat1.de und rtl.de); die andere Gruppe baut ihre Webpräsenz zu regelrechten Informationsportalen aus (z. B. BBC.co.uk; orf.at), deren Inhalte weit über das ausgestrahlte Programm hinausgehen.

Auch wenn Online-Medien (noch) zum geringen Teil aus eigenen Inhalten bestehen, so überwinden sie schon heute mühelos die medialen und kommunikativen Grenzen. Textbasierte Nachrichten werden durch Audio/Videostreaming ebenso ergänzt wie durch das Angebot von thematischen Chats und Foren, die von der Redaktion moderiert werden. Einzelne Online-Medien stellen den Leserinnen und Lesern die generierte Öffentlichkeit zur Verfügung, indem diese online und in Echtzeit für alle anderen lesbare Kommentare zu den redaktionellen Beiträgen publizieren können.

Auf der Stufe der Inhaltegenerierung erweist sich die Vielzahl der neuen Anbieter aber auch als problematisch. So konstatiert Christoph Neuberger, im Internet habe sich „eine Grauzone um den Journalismus herum gebildet“ (Neuberger 2000, 310). In seiner Untersuchung über den Journalismus im Internet ist Neuberger auf Formen von „Scheinjournalismus“ gestoßen, die sich als Gratwanderung zwischen Unternehmenskommunikation und Journalismus erweisen. Durch professionelle optische und gestalterische Aufmachung täuschen manche Online-Medien über die dürftige journalistische Leistung hinweg.

Mit dem Heranreifen der Online-Medien könnte sich diese Gratwanderung als temporäres Phänomen herausstellen. Längerfristig, so ist jedenfalls die bisherige Mediengeschichte zu interpretieren, setzen sich jene medialen Angebote durch, die journalistische Leistung und Glaubwürdigkeit zum zentralen Differenzierungsmerkmal erheben.

Dies kann nur gelingen, wenn die journalistischen Fähigkeiten mit der Produktvielfalt Schritt halten. Online-Journalismus erfordert zusätzliche Fertigkeiten, die über die herkömmliche Qualifikation hinausgehen. Vor allem der Umgang mit dem direkten Publikumsresponse in Form von E-Mail, aber auch in der Form moderierter Diskussionsforen, stellt neue Anforderungen.

Neue Anforderungen werden auch an die Selektionsfähigkeiten von Journalistinnen und Journalisten gestellt. Aus der Masse der im Netz verfügbaren Informationen die relevanten Bestandteile auszufiltern, bedarf spezieller Fertigkeiten. Die Versuchung ist dabei groß, ungeprüft Informationen zu übernehmen und weiterzuverbreiten. Diese jour- 
nalistische Anforderung betrifft Online-Journalismus und herkömmlichen Journalismus gleichermaßen.

Verarbeitung und Organisation: Online-Medien sind fast ausnahmslos privatwirtschaftlich organisiert. Selbst die Online-Ableger öffentlicher Rundfunkanstalten sind vielfach in privatrechtliche Organisationen ausgegliedert (vgl. BBC Online). Auch wenn die Abhängigkeiten von den jeweiligen Stammhäusern vor allem auf der inhaltlichen Ebene weiterhin als erheblich einzustufen sind, so unterliegen Online-Medien dennoch direkt den Marktmechanismen.

Mit dem Grad der Unabhängigkeit der Online-Medien von den Stammhäusern nimmt die Unterordnung dieser Medien unter den ökonomischen Imperativ zu. Börsenkotierte Online-Medien unterziehen sich der Beurteilung durch Analysten, deren Rationalitäten weniger von der publizistischen Relevanz der Inhalte als von dem Börsenwert des Unternehmens gesteuert sind. Konstantin Urban, Bereichsleiter Neue Medien bei der Verlagsgruppe Georg von Holtzbrinck, stellt fest, dass bei Internetunternehmen andere ökonomische Grundsätze gelten. „Bei der Bewertung von Internet-Unternehmen kommen andere Bewertungsmodelle mit anderen Ausprägungen zum Tragen, wie Multiples auf den Umsatz oder die Anzahl der Besucher der Website“ (2000, 2). Solche medienfremden Bewertungsmodelle beschleunigen den Prozess der Ökonomisierung, indem sie publizistische Leistungen als Maßstab ausschließen.

Vertrieb: Online-Medien sind wie alle Medien Informationsgüter. Sie werden digital hergestellt, verarbeitet und ebenso digital verbreitet. Für die Verbreitung sind nicht die realen Kosten für den Aufbau und Betrieb eines elektronischen Netzwerkes zu entrichten. Vielmehr tragen die Nutzerinnen und Nutzer dieser Medien den größten Teil der Vertriebskosten, indem sie an einem der Netze oder Plattformen teilnehmen. Der größte Teil der Vertriebskosten kann von den Medienunternehmen also abgewälzt werden. Dadurch nimmt das von den analogen Medien bekannte Ungleichgewicht zwischen Fixkosten für die Herstellung der „First Copy“ und den variablen Kosten für den Vertrieb weiter zu.

Gleichzeitig nimmt aber auch der Aufwand für die Marktkommunikation zu. Je populärer das Internet insgesamt wird, desto höher werden die Kosten für die Gewinnung neuer Nutzerinnen und Nutzer. Niedrige Transaktionskosten waren für Online-Medien also nur ein temporärer Vorteil, der mit der fortschreitenden Internet-Diffusion in den Haushalten wieder verschwindet. Höhere Marketingkosten werden schon mittelfristig die niedrigen Vertriebskosten wieder kompensieren.

Endgeräte: Die Geräte für den Empfang von Online-Medien sind in aller Regel nicht speziell für diese Nutzungsform ausgelegt. Online-Medien konkurrieren mit einer Vielzahl anderer Inhalte um die Aufmerksamkeit der Nutzerinnen und Nutzer dieser Geräte. Die mangelnde Kontrolle der Medien über die eingesetzten Endgeräte verschärft tendenziell den Wettbewerb. Während gedruckte Zeitungen zumindest auf dem Trägermedium Papier keinem intermediären Wettbewerb ausgesetzt waren, neutralisiert die Technik der digitalen Empfangsgeräte diesen Wettbewerbsvorteil. Online-Medien treten also nicht nur mit anderen (analogen und Online-)Medien in Konkurrenz, sondern bei jedem Nutzungsvorgang zugleich auch mit allen anderen im Internet verfügbaren Inhalten.

Rezeption: Online-Medien erschließen ein bisher nicht bedientes Marktsegment. Sie sind in der Lage, kleine und mittlere Zielgruppen zu bedienen, die bisher aufgrund der hohen Vertriebs- und Logistikkosten für Massenmedien unerreichbar blieben. Solche Nutzergruppen in einer Größenordnung von wenigen hundert bis einigen tausend Personen (Mesomärkte) können nahezu unabhängig von ihrem physischen Aufenthaltsort 
mit medialen Inhalten versorgt werden. Online-Medien beschleunigen den Prozess der Publikumssegmentierung. Solche mesomarktorientierten Online-Medien nehmen die klassische Massenmedienfunktion der Herstellung von Öffentlichkeit kaum noch wahr.

Mit der zunehmenden Diffusion von Internet-Anschlüssen in den Haushalten (in Deutschland hat sich die Zahl der Nutzer zwischen 1997 und 2000 auf 18,3 Mio. vervierfacht; van Eimeren/Gerhard 2000, 339) verschärft sich der Wettbewerb zwischen Online- und Offline-Medien. Bisher konnten allerdings noch keine signifikanten Auswirkungen der Internet-Nutzung auf die sonstigen Mediennutzungsgewohnheiten nachgewiesen werden. Vielmehr blieb beispielsweise die Fernsehnutzung zwischen 1997 und 2000 stabil. „In der direkten Konkurrenz zueinander scheinen Internet und Fernsehen unterschiedliche Bedürfnisse zu befriedigen. Internet entwickelt sich zu einem sehr zielgruppengerichteten Medium, von dem Informationen aus sehr verschiedenen Bereichen nachgefragt werden. Das Fernsehen bleibt offensichtlich das genreübergreifende Leitmedium (...)“(ebd., 346).

Trotz der bisher geringen Substitutionstendenzen analoger Medien durch OnlineMedien ist die Zeit, die den Menschen im Alltag für die Nutzung von Medien zur Verfügung steht, nicht beliebig erweiterbar. Mit steigender Konsumrivalität vor allem zwischen den Online-Medien und anderen Internetinhalten ist daher zu rechnen.

\section{Neue Geschäftsmodelle für Online-Medien}

Verschärfter Wettbewerb, inhalteneutrale Rezeptionsplattformen, verschobene Kostenstrukturen und verändertes Konsumverhalten bei der Nutzung von Online-Medien erzwingen neue Geschäftsmodelle. Als kennzeichnend für Online-Geschäftsmodelle gelten der Einsatz von vielschichtigen Ertragsnetzwerken, die Erschließung von neuen Erlösquellen durch Provisionen und Kommissionen und die Verschmelzung von medialer und individueller Kommunikation. Mit den neuen Erlösmodellen entstehen neue Abhängigkeiten. Diese lassen sich am Beispiel der Provisionen verdeutlichen. Leitet ein Online-Medium Kundschaft an einen Online-Shop weiter und führt diese Weiterleitung zu einem Kaufvorgang, so erhält das Online-Medium einen vereinbarten Prozentsatz des Kaufpreises als Provision. Nimmt diese Finanzierungsform spürbare Größenordnungen an, so hat das Online-Medium alles Interesse daran, die Waren und Dienstleistungen „seiner“ Online-Shops in der Berichterstattung zu begünstigen. Gegenüber der herkömmlichen Werbefinanzierung besteht ein qualitativer Unterschied: Während im Fall der Werbung der erzielbare Werbepreis nach Werbeleistung (Kontakten) bemessen wird, fließen Provisionen erst bei vollzogener Kaufhandlung. Das Risiko der Werbewirkung wird also von den Werbungtreibenden zu den Medien verschoben.

Direkt finanzierte Online-Medien sind große Ausnahmen. Geringe Produktdifferenzierung und nahezu beliebige Verfügbarkeit von Substitutionsprodukten im Internet erschweren die Etablierung von Preisen für die Inhaltenutzung im Massengeschäft. Lediglich in Nischenmärkten, in denen erhöhte Anforderungen an Qualität, Aktualität, Exklusivität oder Verlässlichkeit gelten, können nutzungsbezogene Beiträge realisiert werden.

\section{Ausprägungen der Ökonomisierung}

Wenn als Ökonomisierung der Vorrang nicht-publizistischer Ziele vor publizistischen Zielen verstanden wird, so erweist sich die Ökonomisierung als das dominierende Gestaltungsprinzip der Online-Medien. Die erste der eingangs formulierten Thesen lässt 
sich also bestätigen. Weder Start-up Online-Medien noch ausgelagerte Online-Medien sind einem spezifischen publizistischen Leistungsauftrag verpflichtet. Vielmehr streben Online-Medien sehr pragmatisch nach neuen Funktionalitäten bzw. nach strategischen Allianzen, in die sie attraktive Inhalte einbringen. Durch die große Affinität von OnlineMedien zu den Finanzmärkten entsteht ein Wettbewerbsdruck in Richtung Marktwertsteigerung. Die Erfolgsbemessungsmaßstäbe von Online-Medien sind diejenigen der „New Economy“ und nicht verlegerische publizistische Ziele. Die bisher beobachteten journalistischen Leistungen der Online-Medien bleiben hinter denjenigen der analogen Medien zurück oder übernehmen deren Resultate, ohne diese nachhaltig aufzuwerten. Inhaltebündelung und die Herstellung von rezeptionsgetriebenen Versionen erhöhen Reichweite, Marktanteil und damit den Ökonomisierungsgrad, der Beitrag zur publizistischen Vielfalt ist aber gering.

Die zweite These, wonach der Kommerzialisierungsgrad der Online-Medien denjenigen der analogen Medien mit Ausnahme hochpreisiger Nischenprodukte übersteigt, erscheint ebenfalls plausibel. Online-Medien werden überwiegend über den Vertriebsweg Internet transportiert. Die virtuelle Nähe der Online-Medien zu allen anderen Internet-Inhalten zwingt ihnen deren Regeln auf. Indirekte Erlöse, die auf Provisionen, Werbung und Kommissionen beruhen, bilden die Grundlage der einschlägigen Geschäftsmodelle. In aller Regel folgen Online-Medien dem Geschäftsmodell der indirekten Erlöse und verzichten auf Einnahmen aus dem Verkauf ihrer Dienstleistung. Direkte Erlöse können nur in Einzelfällen erzielt werden. Dies betrifft Angebote mit erhöhten Anforderungen an Aktualität, Qualität, Verlässlichkeit und Exklusivität oder aber die Erfüllung spezieller Wünsche, etwa die Nutzung des Online-Archivs eines Mediums. Die für kostenpflichtige Informationen und Dienstleistungen verlangten Preise werden von Online-Medien im Verhältnis zu ihrer vermuteten Wertigkeit festgesetzt, was je nach Exklusivität der Information das Preisniveau steigert.

Auch die dritte These, wonach die Marktmacht führender Marktteilnehmer auch nach dem Auftreten der Online-Medien weiter zunimmt, erscheint plausibel. Im Gegensatz zu den klassischen analogen Medien, die im deutschen Sprachraum in der Regel im Eigentum von untereinander mehr oder weniger verflochtenen Medienhäusern stehen, weisen Start-up Online-Medien eine deutlich vielfältigere Eigentümerstruktur auf. Sie konkurrieren eher untereinander als mit den klassischen Medien und legen eher Analysten gegenüber Rechenschaft ab als einer einzelnen Konzernleitung. Ihr mehrschichtiges Kerngeschäft (Medium, Interaktionsplattform, Aufmerksamkeitsvermittlung) lässt Online-Medien sowohl für Medienunternehmen als auch für Unternehmen der Telekommunikations- und der Informatikbranche als Partner attraktiv erscheinen. Heterogen zusammengesetzte Joint Ventures großer Konzerne sorgen einerseits für eine großzügige finanzielle Ausstattung (was sich in intensiver Marktbearbeitung bemerkbar macht), stellen andererseits aber auch hohe Markteintrittsbarrieren für neue Wettbewerber dar. Erfolgreiche Online-Medien hatten bisher kaum die Chance, aus eigener Kraft zu relevanten Wettbewerbern heranzuwachsen. Vielmehr wurden solche Start-ups schon in der Expansionsphase von mehr oder weniger branchenfremden Investoren übernommen. Diese Entwicklung führt zu einem hoch konzentrierten Wettbewerb unter einer relativ kleinen Anzahl von Teilnehmern mit überdurchschnittlicher Marktmacht.

\section{Fazit}

Online-Medien treten in heterogener Form in die Medienmärkte ein, wobei nur ein Teil von ihnen die klassischen Anforderungen an Massenmedien erfüllt. Den höchsten Pro- 
fessionalitätsgrad weisen diejenigen Online-Medien auf, die direkt oder indirekt mit bestehenden Medien verbunden sind. Unabhängige sowie mit branchenfremden Unternehmen verbundene Start-up Online-Medien bilden ein neues Phänomen, dessen spezifische Merkmale sich noch in der Ausprägungsphase befinden.

Der überwiegende Teil der bisher etablierten Online-Medien leistet im Vergleich zu den bestehenden analogen Medien einer mehr oder weniger deutlich ausgeprägten inhaltlichen Ausdünnung Vorschub. Dies äußert sich entweder in einem reduzierten inhaltlichen Umfang der Online-Ausgabe gegenüber der analogen Ausgabe oder im Auftreten von „Scheinjournalismus“, der sich in der kaum redaktionell bearbeiteten Wiedergabe von anderswo generierten Inhalten erschöpft.

Die Organisationsform der Online-Medien als eigenständige Profitcenter bestehender Unternehmen (medialer oder medienfremder Provenienz) begünstigt die Fokussierung auf marktliche Ziele. Mit der Nähe zu den Finanzierungsgrundlagen der „New Economy“ durch Venture Capital nimmt der Druck zur Erwirtschaftung überdurchschnittlicher Renditen zu.

Ebenso verschärft die gelungene Abwälzung der Vertriebskosten der Online-Medien auf die Nutzerinnen und Nutzer den von den analogen Medien hinlänglich bekannten Effekt zur Unternehmenskonzentration, weil in gesättigten Märkten bei hohen Fix- und geringen variablen Kosten der Monopolist am effizientesten wirtschaftet.

Die Finanzierungsbasis der Online-Medien schließlich besteht nahezu zur Gänze aus indirekten Erlösen. Die direkte Zahlungsbereitschaft von Nutzerinnen und Nutzern lässt sich bislang nur in kleinen Marktnischen abschöpfen und kommt für die Finanzierung von Online-Medien mit massenmedialem Anspruch nicht in Betracht. Die technisch ermöglichte Zusatzfinanzierung durch die Erzielung von Provisionen bei vollzogener Kaufhandlung von weitergeleitetem „Traffic“ verschärft die als Kommerzialisierung beschriebene Problematik.

Die eingangs aufgeworfene Frage, ob durch die Digitalisierung die Tendenzen der Ökonomisierung und Kommerzialisierung eine Beschleunigung erfahren, ist im Hinblick auf die Strukturen der bisher in den Medienmarkt eingetretenen Online-Medien zu bejahen.

\section{Literatur}

Heinrich, Jürgen (1994): Medienökonomie Band 1: Mediensystem, Zeitung, Zeitschrift, Anzeigenblatt. Opladen.

Heinrich, Jürgen (1999): Medienökonomie Band 2: Hörfunk und Fernsehen. Opladen.

Neuberger, Christoph (2000): Journalismus im Internet: Auf dem Weg in die Eigenständigkeit? In: Media Perspektiven H. 7, S. 310 - 318.

Sennwald, Nicola (1998): Massenmedien und Internet. Zur Marktentwicklung in der Pressebranche. Wiesbaden.

Shapiro, Carl; Varian, Hal (1998): Information Rules. A Strategic Guide to the Network Economy. Harvard.

Urban, Konstantin (2000): Fremde Welten. Weshalb sich klassische Verlagsunternehmen mit der Internet-Ökonomie schwertun. In: Handelsblatt Nr. 69 vom 6. April 2000.

van Eimeren, Birgit; Gerhard, Heinz (2000): ARD/ZDF-Online-Studie 2000: Gebrauchswert entscheidet über Internetnutzung. In: Media Perspektiven H. 8, S. $338-349$.

Zerdick, Axel; Picot, Arnold; Schrape, Klaus u. a. (1999): Die Internet-Ökonomie. Strategien für die digitale Wirtschaft. Heidelberg/New York.

Zürn, Matthias (2000): Print- und Onlinezeitungen im Vergleich. In: Media Perspektiven H. 7, S. $319-325$. 